\title{
CRIMSONpublishers
}

http://www.crimsonpublishers.com

Opinion

Exp Tech Urol Nephrol

ISSN 2578-0395

\section{Current Concepts and Controversies Regarding Surgery for Male Infertility}

\author{
Marcelo Rodríguez Peña* \\ Department of Urology, Military Hospital Campo de Mayo, Argentina
}

*Corresponding author: Marcelo Rodríguez Peña, Sexual and Reproductive Medicine Program, Military Hospital Campo de Mayo, Holanda 7895 (CP 1682), Buenos Aires, Argentina

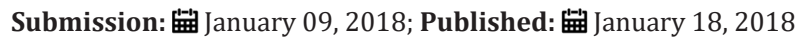

Keywords: Epididymostomy; Intracitoplasmic; varicocele; lithiasis

Abbreviations: ICSI: Intracitoplasmic Sperm Injection; micro-TESE: Microsurgical Testicular Biopsy; TESE: Testicular Biopsy

\section{Opinion}

The diagnostic and therapeutic approach of the infertile man has suffered in recent times spectacular changes derived from the development of complex assisted reproduction techniques such as the possibility of getting gestations with a single sperm injected into the interior of the oocyte. This technique is popularly known as ICSI (intracitoplasmic sperm injection). Due to this revolutionary technique, many paradigms of reproductive medicine were modified and the question arises: Why, under this reality, is necessary the evaluation and treatment of the infertile male? And even more: Why should we propose surgical treatments to solve certain conditions of male infertility if we could resort directly to the ICSI technique?

The answer is the following:

i. Most couples prefer to achieve pregnancy as naturally as possible. The treatment of man can allow natural conception in a percentage of them.

ii. Pregnancy rate by using ICSI in male factor are low (15$18 \%$ per cycle) if we compare it with the one obtained when we perform this technique by female factor (25-30\% per cycle) [1-3].

Many current studies conclude that andrological treatments (pharmacological or surgical) not only improve the seminal quality to achieve spontaneous pregnancy but also improve the quality of the gametes to be used in later ICSI treatments. In this last aspect the surgical treatment of the infertile man acquires a fundamental importance. The microsurgical vaso-vasostomy results in the appearance of sperm in the ejaculate in more than $90 \%$ of patients and also in $50-80 \%$ of spontaneous pregnancies in their partners, of course, depending on the age and the co-existence of some associated pathology in them [4].
Epididymal obstruction requires a microsurgical

vasoepididymostomy. This surgical technique is highly effective in terms of achieving the appearance of gametes in the semen. In the case that spontaneous pregnancies are not achieved with these procedures but sperm cells appear in the semen, even in a small amount, the use of it in ICSI techniques is an alternative [5]. Surgical techniques to treat varicocele are highly safe and effective. There is a significant improvement in seminal quality in $70 \%$ of operated patients and in pregnancy rates in their partners if they have not a concomitant female factor [6-8].

Although surgery for male infertility has historically been developed by specialists in urology, surgical procedures for infertility in men are very rare in urological services for several reasons:

i. Few services have urologists specialized in infertility who work in coordination with centers of reproductive medicine.

ii. The programs of urological residences generate little emphasis in the study and treatment of the infertile man in contrast with other subspecialties (for example, lithiasis, urological oncology, urogynecology, etc.)

iii. It requires a very specialized surgical training (especially in microsurgery) and a prolonged surgical work (usually several hours) and coordination with the semen and embryology laboratory that must receive samples of testicular tissue or seminal fluid to analyze and cryopreserve.

\section{Conclusion}

Microsurgical reconstruction or transurethral resection of veromontanum remains the best treatment for men with obstructive azoospermia susceptible to repair. The techniques of 
sperm retrieval associated with ICSI are the best alternative in those patients where surgical repair of the seminal path is not feasible. It is now widely demonstrated that varicocelectomy improves the seminal quality and the chances of pregnancy in males with mild and moderate seminal alterations and the quality of the gametes to be used in an ICSI technique in patients with severe seminal alterations. Microsurgical varicocelectomy is the procedure of choice due to the low rate of complications and rapid recovery of patients operated with this technique.

In men with secretory azoospermia, testicular biopsy (TESE) associated with the ICSI technique has achieved encouraging results. Microsurgical testicular biopsy (micro-TESE) has been shown to be more efficient than TESE in terms of sperm recovery rate but has the disadvantage of being a more damaging technique for the gonad, not ruling out the possibility of postoperative hypogonadism if it is applied to both gonads [9]. The current knowledge in molecular biology and immunology associated with the principles of microsurgery could allow us to develop, in the near future, adequate tools to "signalize" the scarce and elusive foci of spermatogenesis with minimal surgical trauma to the gonadal parenchyma [9].

\section{References}

1. Tucker MJ, Morton PC, Wright G, Ingargiola PE, Jones AE, et al. (1995) Factors affecting success with intracytoplasmic sperm injection. Reprod Fertil Dev 7(2): 229-236.
2. Schwarzer JU, Fiedler K, Hertwig IV, Krusmann G, Würfel W, et al. (2003) Male factors determining the outcome of intracytoplasmic sperm injection with epididymal and testicular spermatozoa. Andrologia 35(4): 220-226.

3. Osmanagaoglu K, Vernaeve V, Kolibianakis E, Tournaye H, Camus M, et al. (2003) Cumulative delivery rates after ICSI treatment cycles with freshly retrieved testicular sperm: a 7-year follow-up study. Hum Reprod 18(9): 1836-1840.

4. Monseny JMP (2012) Patología obstructiva de la vía seminal. In: Tratado de Andrología y Medicina Sexual. Ed. Panamericana, pp. 355-367.

5. Lee R, Li PS, Goldstein M, Tanrikut C, Schattman G, et al. (2008) A decision Analysis of treatments for obstructive azoospermia. Hum Reprod 23(9): 2043-2049.

6. Coccuza M, Cocuzza MA, Bragais FM, Agarwal A (2008) The role of varicocele repair in the new era of assisted reproductive technology. Clinics 63(3): 395-404.

7. Khera M, Lipshultz Ll (2008) Evolving approach to the varicocele. Urol Clin North Am 35(2): 183-189.

8. Peña MR, Alescio L, Russell A, Lourenco JM, Alzu G, et al. (2009) Predictors of improved seminal parameters and fertility after varicocele repair in Young adults. Andrologia 41(5): 277-281.

9. Smith RP, Lowe GJ, Kavoussi PK, Steers WD, Costabile RA, et al. (2012) Confocal fluorescence microscopy in a murine model of microdissection testicular sperm extraction to improve sperm retrieval. J Urol 187(5): 1918-1923. 